\title{
Three dimensional global test particle simulation of cosmic ray acceleration and escape in supernova remnants
}

\section{Shoma Kamijima*}

Aoyama Gakuin University

E-mail: shoma@phys.aoyama.ac.jp

\section{Yutaka Ohira}

The University of Tokyo

E-mail: y.ohira@eps.s.u-tokyo.ac.jp

\begin{abstract}
The origin of cosmic rays (CRs) is a long standing problem in astrophysics. CRs spectrum breaks at $10^{15.5} \mathrm{eV}$ which is called the knee energy. A perpendicular shock acceleration has been considered an efficient acceleration mechanism candidate to reach CRs up to the knee energy. it is suggested that the escape process is important to determine the maximum energy and a CRs spectral index. However, the escape process from a perpendicular shock region is still unknown. In this study, we investigate the acceleration time at perpendicular shock and the escape process from this region. Using a test particle simulation, we shows the rapid acceleration at the perpendicular shock and that the diffusion approximation is not valid in the case that the magnetic fluctuation is weak in an upstream stream region and is strong in a down stream region. In addition, we perform the three dimensional global test particle simulation to investigate the escape process from a perpendicular shock region. We will perform more simulations in the cases of other parameters to understand the escape from a perpendicular shock region.
\end{abstract}

36th International Cosmic Ray Conference -ICRC2019-

July 24th - August 1st, 2019

Madison, WI, U.S.A.

${ }^{*}$ Speaker. 


\section{Introduction}

The origin of cosmic rays (CRs) is a long standing problem in astrophysics. In the standard model, Galactic CRs below $10^{15} \mathrm{eV}$ (knee energy) are accelerated by the diffusive shock acceleration (DSA) in Galactic supernova remnants (SNRs). In a parallel shock region, CRs easily move along a magnetic field line and diffuse to the far upstream region. On the other hand, in a perpendicular shock region, CRs cannot diffuse to the far upstream region compared with the parallel shock case because it is hard for CRs to diffuse perpendicular to the magnetic field line. As a result, the acceleration time in the perpendicular shock becomes smaller than that in the parallel shock [7]. Therefore, the perpendicular shock region has been considered as an efficient accelerator like PeVatron.

There are several problems for the acceleration in the perpendicular shock. One is the injection problem. The injection to DSA is expected to be efficient for parallel shocks because the perpendicular magnetic field prevents CRs from returning back to the upstream region [8]. Caprioli \& Spitkovsky (2014) [10] showed by hybrid simulations that no particles are injected to DSA from thermal particles at the perpendicular shock propagating to a fully ionized plasma. On the other hand, Ohira (2016) [1] recently showed by three-dimensional hybrid simulations that particles are injected to DSA at the perpendicular shock in a partially ionized plasma, so that particles are rapidly accelerated by DSA. A necessary and sufficient condition for the injection to DSA at the perpendicular shock is an open question.

Another problem for the acceleration in the perpendicular shock is the spectral index of the accelerated particle. If a fluctuated component of the magnetic field, $\delta B$, is comparable to or larger than an uniform component of the magnetic field, $B_{0}$, the particle diffusion becomes isotropic, so that the acceleration time scale at the perpendicular shock become identical to that at the parallel shock. The rapid acceleration at the perpendicular shock requires a small amplitude of the fluctuated component, $\delta B / B_{0} \ll 1$. It is shown that if the magnetic field fluctuation is weak in the upstream and downstream regions, energy spectra of accelerated particle at the perpendicular shock becomes steeper than expected from observations [11]. However, some observations [12,2] and simulations $[14,13,9,3]$ suggest that the magnetic field is strongly amplified in the downstream region, that is, $\delta B / B_{0}>1$. Therefore, there is a possibility of $\delta B / B_{0} \ll 1$ in the upstream region and $\delta B / B_{0}>1$ in the downstream region. In this work, we first investigate the particle acceleration in that case.

There has been an increasing interest in escape of CRs from SNRs because the escape process of CRs changes the energy spectrum of CRs [4] and is important for understanding gamma-ray spectra from middle-aged SNRs [5]. The maximum energy of CRs and its evolution are determined by the escape process [15]. Although the time evolution of the maximum energy of CRs has been investigated by several papers [16, 5], they assumed isotropic diffusion or the parallel shock acceleration. As the second topic in this work, we investigate the escape of CRs from the perpendicular shock region of SNRs. It is still unknown how CRs escape from a supernova remnant after acceleration at the perpendicular shock. 


\section{Simulation}

We need to take account into the shape of a system to investigate the escape of CRs from the perpendicular shock region because CRs cannot escape to the upstream region of a PLANE perpendicular shock. Furthermore, in order to solve the drift acceleration at the perpendicular shock, we cannot use a diffusion approximation for the CR motion in the upstream region. Therefore, we perform three-dimensional global test-particle simulations in this work.

In the shock upstream region, the magnetic field is given by the sum of a uniform magnetic field, $\vec{B}_{0}=B_{0} \vec{e}_{z}$ and a static turbulent magnetic field, $\delta \vec{B}(x, y, z)$, which is given by a summation of many transverse waves [17]. The isotropic Kolmogorov spectrum, $G(k)$, is assumed for the turbulent magnetic field as follows.

$$
G(k)=\frac{\left(\frac{k}{k_{0}}\right)^{3}}{\left[1+\left(\frac{k}{k_{0}}\right)^{2}\right]^{7 / 3}},
$$

where $k$ is the wave number and the spectrum has a peak at $k_{0}$.

For particles in the shock upstream region, we solve the equation of motion in the magnetic field given by the above. In the shock downstream region, we assume that particles are scattered isotropically in the fluid rest frame and the diffusion is the Bohm diffusion in the magnetic field of $100 B_{0}$.

\section{Simulation for plane shock with a constant velocity}

We first investigate the particle acceleration at the perpendicular shock, where $\delta B / B_{0} \ll 1$ in the upstream region and $\delta B / B_{0} \gg 1$ in the downstream region. We here consider a plane shock front propagating with the shock velocity of $u_{\mathrm{sh}}$ in the upstream rest frame. Simulation parameters in this work are as follows. The shock velocity and the shock compression ratio are $u_{\mathrm{sh}}=0.01 \mathrm{c}$ and $r=4$, where $c$ is the speed of light. The upstream magnetic fluctuations is $\delta B / B_{0}=0.1$. The peak, minimum, and maximum wave numbers of the magnetic field fluctuation are given by $k_{0}=2 \pi \times 10^{-5} r_{\mathrm{g}, 0}^{-1}, k_{\min }=0.1 k_{0}$, and $k_{\max }=10^{7} k_{0}$, where $r_{\mathrm{g}, 0}$ is the initial gyroradius of the injected particle. The time step and the number of waves to describe the magnetic field fluctuation are $\Delta t=0.01 r_{\mathrm{g}, 0} / c$ and $N=320$, respectively. We solve the equation of motion of simulation particles in the upstream region, but we solve the random walk in the downstream region. We assume the Bohm scattering in the magnetic field of $100 B_{0}$. Then, the scattering time in a shock downstream region is given by $t_{\mathrm{sc}}=\Delta t\left(p / p_{0}\right)$, where $p / p_{0}$ is the momentum normalized by the initial momentum. Simulation particles are impulsively injected at the initial time on the shock surface.

Figure 1 shows the acceleration time as a function of the normalized momentum, $p / p_{0}$. The black points show the simulation result. The red line shows the acceleration time of DSA when the residence time in the shock upstream region is given by one gyro period. The blue line shows the acceleration time of DSA for the Bohm diffusion in $B=B_{0}, t_{\mathrm{acc}} \approx 4 D_{\mathrm{Bohm}} / u_{\mathrm{sh}}^{2}$. The green line shows the acceleration time of DSA for the perpendicular diffusion, $t_{\mathrm{acc}} \approx 4 D_{\text {perp }} / u_{\mathrm{sh}}^{2}$ and 


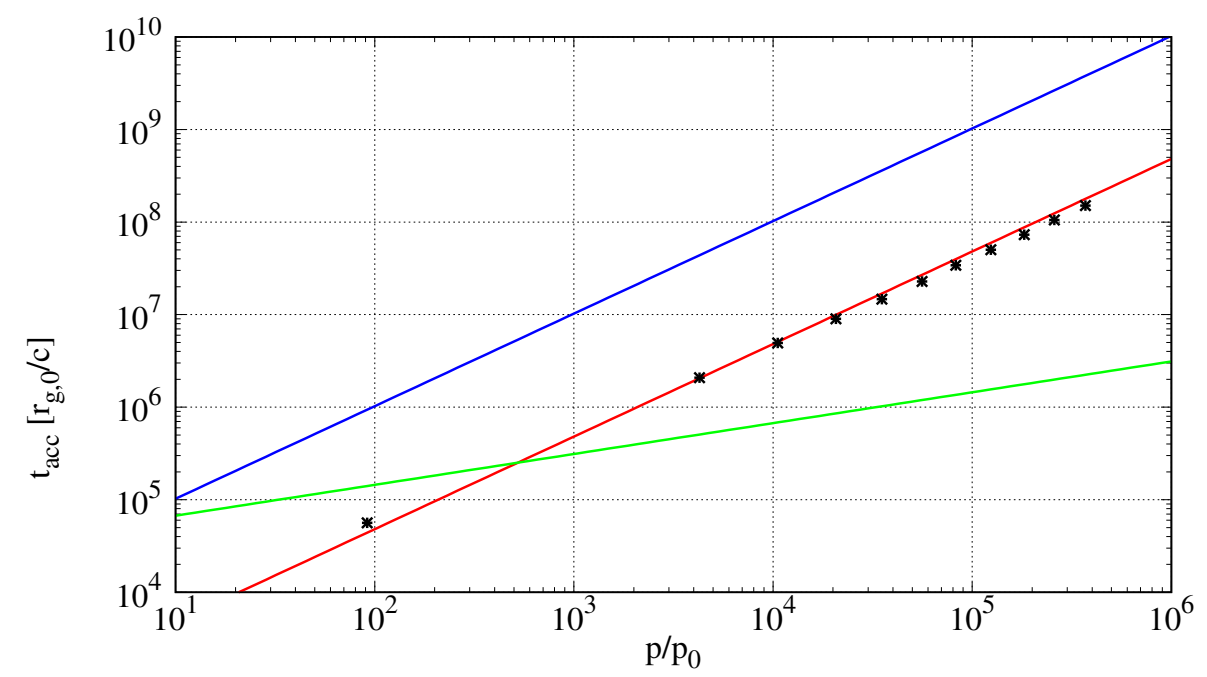

Figure 1: Acceleration time at the perpendicular shock for $\delta B / B_{0}=0.1$ in the upstream region and $\delta B / B_{0}=$ 100 in the downstream region. The black points show the simulation result. The three lines show the theoretical acceleration times. See text for details.

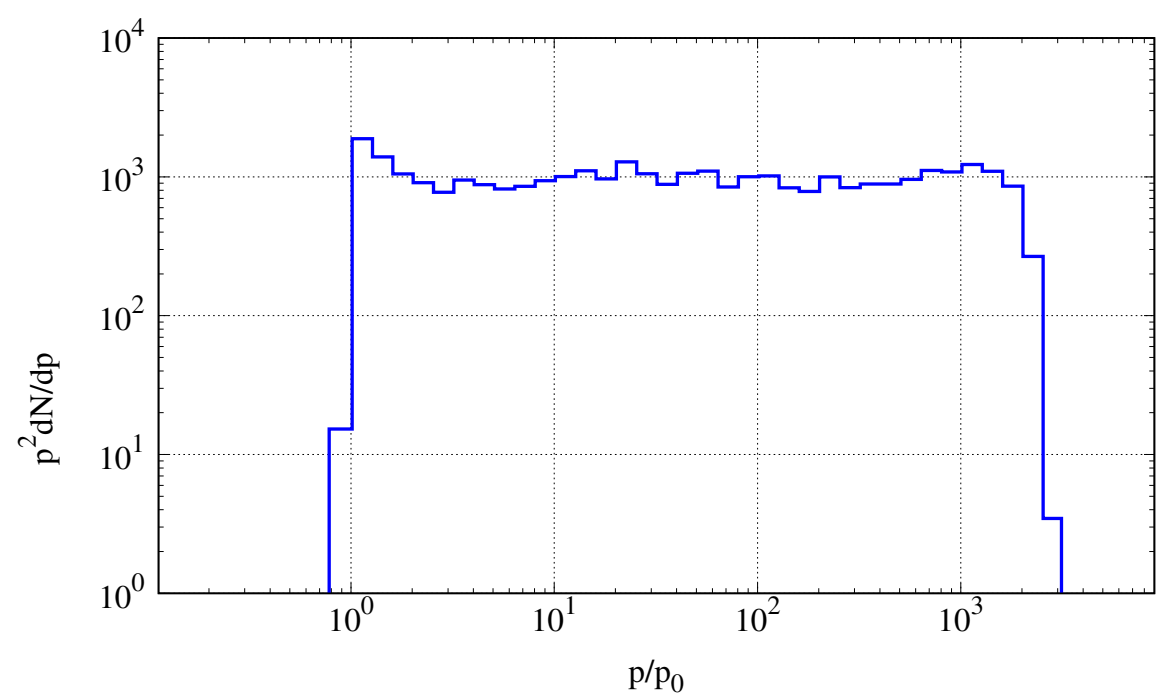

Figure 2: Energy spectrum of all simulation particles at $t=5.91 \times 10^{6} r_{\mathrm{g}, 0} / c$. 
$D_{\text {perp }} \approx 10^{-5} D_{\text {para }}[18]$. For all the acceleration time of DSA, we neglect the downstream residence time. Our simulation shows the rapid acceleration at the perpendicular shock and that the diffusion approximation is not valid in this case.

Figure 2 shows the energy spectrum of all particles at $t=5.91 \times 10^{6} r_{g, 0} / c$. The energy spectrum becomes $d N / d p \propto p^{-2}$. Therefore, our simulation shows that the rapid acceleration at the perpendicular shock is compatible with the canonical energy spectrum of $p^{-2}$.

\section{Simulation for a global supernova remnant shock}

We investigate the escape process from a perpendicular shock region of a supernova remnant by using a three-dimensional global test particle simulation. The supernova remnant is assumed to be a spherically symmetric system, so that the shock velocity and the fluid velocity in the shocked region have only the radial component. The evolution of the shock velocity, radius and fluid velocities in all region are given by the analytical models $[19,6]$. In this simulation, we set $\delta B / B_{0}=1$ in the upstream region of the forward shock, $\delta B / B_{0}=100$ in a downstream region, and $B=0$ in the freely expanding ejecta. We solve the equation of motion of simulation particles in the upstream region of the forward shock, but we solve the random walk in the downstream region. We assume the Bohm scattering in the magnetic field of $100 B_{0}$. The particle motion in the freely expanding ejecta is free streaming. We perform this simulation from $0.1 t_{\text {Sedov }}$ to $1 t_{\text {Sedov }}$, where $t_{\text {Sedov }}=200 \mathrm{yr}\left(\mathrm{E}_{\mathrm{SN}} / 10^{51} \mathrm{erg}\right)^{-1 / 2}\left(\mathrm{M}_{\mathrm{ej}} / 1 \mathrm{M}_{\odot}\right)^{5 / 6}\left(\mathrm{n}_{0} / 1 \mathrm{~cm}^{-3}\right)^{-1 / 3}$ is the free expansion time scale. $E_{\mathrm{SN}}, M_{\mathrm{ej}}$ and $n_{0}$ are the supernova explosion energy, ejecta mass and ambient number density, respectively.

The initial energy of the injected particles is, $E_{0}=100 \mathrm{GeV}$. The peak, minimum, and maximum wave numbers of the magnetic field fluctuation are given by $k_{0}=2 \pi \times 10^{-3} r_{\mathrm{g}, 0}^{-1}, k_{\min }=0.1 k_{0}$, and $k_{\max }=10^{5} k_{0}$, where $r_{\mathrm{g}, 0}$ is the gyro radius of the injected particle. The time step and the number of waves to describe the magnetic field fluctuation are $\Delta t=0.01 r_{\mathrm{g}, 0} / c$ and $N=240$, respectively. The particles are injected only at the initial time on the equatorial plane of the forward shock surface.

Figure 3 is a snapshot at $0.5 t_{\text {Sedov }}$ projected to yz plane. The horizontal axis and vertical axis are normalized by the initial forward shock radius. The points show all simulation particles. The blue circle shows the forward shock front. The cyan circle shows the reverse shock front. The color of the points shows the particle energy normalized by the initial particle energy, $E_{0}$. We are going to analyze in details and perform more simulations for other parameter cases in future work. 


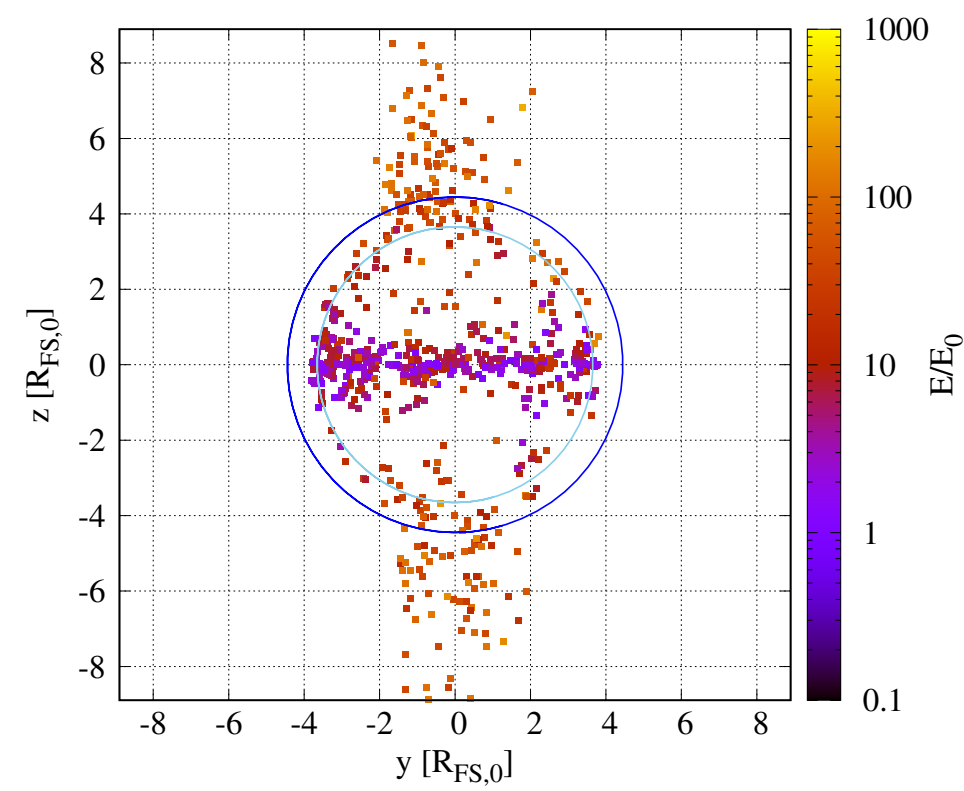

Figure 3: The snapshot at $t=0.5 t_{\text {Sedov }}$ projected to yz plane. The horizontal axis and vertical axis are normalized by the initial forward shock radius. The points show simulation particles. The blue and cyan circles show the forward and reverse shock fronts, respectively. The color of the points shows the particle energy normalized by the initial particle energy, $E_{0}$.

\section{Discussion}

We have performed two simulations. One is the plane shock case to investigate the acceleration time at a perpendicular shock. The other is the supernova remnant shock case to investigate the escape process from a perpendicular shock region. The simulation about the plane shock case shows the rapid acceleration at the perpendicular shock and that the diffusion approximation is not valid in this case. In addition, the energy spectra agrees with the DSA prediction. We perform the three dimensional global test particle simulation. We are going to analyze the supernova remnant shock case in details and perform more simulations for other parameter cases.

\section{Acknowledgements}

We thank R. Yamazaki and S. Tanaka for valuable comments. Numerical computations were carried out on Cray XC50 at Center for Computational Astrophysics, National Astronomical Observatory of Japan. S.K. is supported by Yoshida Scholarship Foundation. Y.O. is supported by MEXT/JSPS Leading Initiative for Excellent Young Researchers. This work is supported by JSPS KAKENHI Grant Number JP16K17702 (Y. O.) and JP19H01893(Y. O.). 


\section{References}

[1] Y. Ohira, Injection to Rapid Diffusive Shock Acceleration at Perpendicular Shocks in Partially Ionized Plasmas, ApJ, 827, 36, 2016

[2] Y. Ohira and Yamazaki, Inverse Compton emission from a cosmic-ray precursor in RX J1713.7-3946, JHEAp, 13, 17, 2017

[3] Y. Ohira, Magnetic Field Amplification by Collisionless Shocks in Partially Ionized Plasmas, ApJ, 817, 137, 2016

[4] Y. Ohira, K. Murase, and R. Yamazaki, Escape-limited model of cosmic-ray acceleration revisited, $A \& A$ 513, A17, 2010

[5] Y. Ohira, K. Murase, and R. Yamazaki, Gamma-Rays from Molecular Clouds Illuminated by Cosmic Rays Escaping from Interacting Supernova Remnants, MNRAS, 410, 1577, 2011

[6] Y. Ohira, S. Kisaka and R. Yamazaki, Pulsar Wind Nebulae inside Supernova Remnants as Cosmic-Ray PeVatrons, MNRAS, 478, 926, 2018

[7] J. R. Jokipii, Rate of Energy Gain and Mamimum Energy in Diffusive Shock Acceleration, ApJ, 313 , 842,1987

[8] D. C. Ellison, M. G. Baring and F. C. Jones, Acceleration Rates and Injection Efficiencies in Oblique Shocks, ApJ, 453, 873, 1995

[9] D. Caprioli and A. Spitkovsky, Cosmic-Ray-induced Filamentation Instability in Collisionless Shocks, ApJ, 765, 20, 2013

[10] D. Caprioli and A. Spitkovsky, Simulations of Ion Acceleration at Non-relativistic Shocks. I. Acceleration Efficiency, ApJ, 783, 91, 2014

[11] M. Takamoto and J. G. Kirk, Rapid Cosmic-ray Acceleration at Perpendicular Shocks in Supernova Remnants, ApJ, 809, 29, 2015

[12] A. Bamba, R. Yamazaki, M. Ueno and K. Koyama, Small-Scale Structure of the SN 1006 Shock with Chandra Observations, ApJ, 589, 827, 2003

[13] T. Inoue, R. Yamazaki and S. Inutsuka, Turbulence and Magnetic Field Amplification in Supernova Remnants: Interactions Between a Strong Shock Wave and Multiphase Interstellar Medium ApJ, 695, 825,2009

[14] J. Giacalone and J. R. Jokipii, Magnetic Field Amplification by Shocks in Turbulent Fluids ApJ, 663, 41,2007

[15] V. S. Ptuskin and V. N. Zirakashvili, Limits on diffusive shock acceleration in supernova remnants in the presence of cosmic-ray streaming instability and wave dissipation ApJ, 403, 1, 2003

[16] V. S. Ptuskin, V. N. Zirakashvili and E, Seo, Spectrum of Galactic Cosmic Rays Accelerated in Supernova Remnants ApJ, 718, 31, 2010

[17] M. Hussein and A. Shalchi, Detailed Numerical Investigation of the Bohm Limit in Cosmic Ray Diffusion Theory ApJ, 785, 31, 2014

[18] J. Giacalone and J. R. Jokipii, The Transport of Cosmic Rays across a Turbulent Magnetic Field ApJ, 520, 204, 1999

[19] C. F. Mckee and J. K. Truelove, Explosions in the interstellar medium PhR, 256, 157, 1995 